

\title{
NUEVAS FUENTES \\ DOCUMENTALES PARA EL \\ ESTUDIO DE LA PRENSA EN \\ ESPAÑA DURANTE EL \\ FRANQUISMO: EL ARCHIVO \\ REGIONAL DE LA COMUNIDAD \\ DE MADRID
}

New sources of information to study Spanish press history during francoism: The Archivo Regional de la Comunidad de Madrid

DOI: http://dx.doi.org/10.12795/RiCH.2021.i17.11

María del Pilar Solís Herráiz

Archivo Regional de la Comunidad de Madrid, España

pilar.solis@madrid.org

ORCID (D) 0000-0002-8707-6387 
Resumen: El objeto de este artículo es dar a conocer los fondos custodiados en el Archivo Regional de la Comunidad de Madrid susceptibles de ser fuente primaria para la investigación en el campo de la comunicación, concretamente en la historia de la prensa en España, en el periodo cronológico que coincide con el franquismo. Estos fondos son "Prensa del Movimiento de Madrid" y "Lucio del Álamo", quien ocupó entre otros cargos el de delegado nacional de Prensa, ambos recientemente tratados (organizados, clasificados, ordenados y descritos) por la Unidad de Descripción del Archivo Regional de la Comunidad de Madrid y puestos a disposición de los investigadores. A través de ellos podemos conocer aspectos tan distintos como la distribución de los ejemplares, el reparto del papel en años de fuertes restricciones (materia prima básica para la prensa), las retribuciones a los distintos colaboradores, el trabajo en los talleres, la incautación de periódicos, inmuebles o maquinaria, etc., en su mayoría de la Prensa del Movimiento $y$, en menor medida, de otros medios de comunicación.

Palabras clave: prensa, franquismo, fuentes, archivos, documentos, Prensa del Movimiento.

\begin{abstract}
The aim of this article is to make a presentation of the documentary collections that are currently held at the Archivo Regional de la Comunidad de Madrid (Spain) susceptible of being an important source of information for researchers, specially for those who study the spanish press history during Francoism. These fonds are "Prensa del Movimiento de Madrid" and "Lucio del Álamo" who was the head of "Delegación Nacional de Prensa" and had other interesting job positions in the media sector. These documents have been identified, organized and described by the Description Unit of the Archivo Regional de la Comunidad de Madrid and, as a consequence, they can be consulted. They show us very useful and interesting information about different topics (the distribution of copies; the delivery of paper, which is basic for a newspaper; payments; workshops; the seizure of newspapers, buildings and machines; etc.) not only about "Prensa del Movimiento" but also about other spanish media.
\end{abstract}

Keywords: press, research sources, archives, archival document, documentary collections, Francoism, Movement's press.

\title{
Introducción
}

La investigación en Historia de la Comunicación tiene un importante recurso en aquellos documentos custodiados en los archivos. Son fuente casi inagotable de datos. Es por ello que los archivos cobran su sentido, para facilitar esas fuentes a los investigadores.

Justino Sinova en una de las primeras publicaciones sobre la censura en la prensa en España durante el franquismo acierta a señalar:

Fui reuniendo toda la información a la que podía tener acceso. El fruto, sin embargo, era insuficiente. Había que llegar a las fuentes (...) Pasé muchas e interesantes horas en el Archivo General de la Administración (...) buceando en 
una documentación que, en gran parte, por primera vez era examinada por ojos ajenos. (Sinova Garrido, 1989: 14-15).

Por ello es importante difundir nuevas fuentes como las actualmente disponibles en el Archivo Regional de la Comunidad de Madrid.

Es en los documentos producidos por la propia institución donde vamos a encontrar los datos que reconstruyan lo sucedido. La labor de tratamiento archivístico, organización, clasificación es fundamental para que esos documentos puedan salir a la luz a través de investigaciones serias y fundamentadas.

\section{Introducción a la Prensa del Movimiento y su contexto}

La prensa en España después de la Guerra Civil se podría configurar en prensa privada y prensa estatal. Dentro de los primeros encontramos medios como $A B C \circ Y A$. Por otro lado, dentro de los segundos, se consideran los que cuentan con el aparato gubernamental y cuyo objetivo es servir al Estado. Dentro de este grupo se encuentra el diario vespertino Pueblo, adscrito al Sindicato Vertical y sobre todo la Prensa del Movimiento.

Hemos de recordar que la denominada Prensa del Movimiento fue el grupo periodístico más importante de España (Reig y Langa, 2011: 165) durante los casi cuarenta años de dictadura de Francisco Franco, que incluía tanto diarios como revistas, semanarios... todos gestionados a través de la Delegación Nacional de Prensa. Por lo tanto, acceder a estas fuentes es tener acceso a la información sobre la mayor parte de la prensa en España en este periodo, sobre todo durante el primer franquismo.

El origen de lo que habría de ser conocido como Prensa del Movimiento se produce durante la Guerra Civil, cuando las fuerzas sublevadas en su avance territorial se hacen con el control de numerosos diarios, editoriales y rotativas pertenecientes al bando enemigo.

Por Ley de 13 de julio de 1940 se entrega la propiedad de todo este conjunto de bienes incautados a Falange Española Tradicionalista y de las JONS (FET y de las JONS), pasando a control de la Delegación Nacional de Prensa y Propaganda ${ }^{1}$.

Desde ese momento el Estado pasa a gestionar alrededor de cuarenta diarios que, configurados bajo el nombre de Prensa del Movimiento, constituiría el grupo

1 En sus diferentes denominaciones orgánicas a lo largo del periodo. 
empresarial más importante del Estado franquista. Además, disponía de una editorial Ediciones del Movimiento-, y de una agencia de noticias -Pyresa (Prensa y Radio Española)-, propias.

Pocos son los archivos en España que han podido poner a disposición de los investigadores documentos producidos por la denominada Prensa del Movimiento. El más importante de ellos, por la amplitud de sus fondos, es el Archivo General de la Administración. Contiene entre ellos el de "Medios de Comunicación Social del Estado", organismo autónomo creado mediante Real Decreto 708/1977, de 15 de abril, para gestionar ese conglomerado de medios durante la democracia.

Otros archivos son los históricos provinciales. Algunos custodian fondos propios de cabeceras de medios de su provincia pertenecientes a la Prensa del Movimiento. Este es el caso del Archivo Histórico Provincial de Cádiz que conserva entre sus fondos los del diario de Jerez de la Frontera La Voz del Sur, el Diario Odiel en el Archivo Histórico Provincial de Huelva o el periódico Nueva España conservado en el Archivo Histórico Provincial de Huesca.

El Archivo Regional de la Comunidad de Madrid cuenta entre sus fondos con dos, que, por sus características, pueden ser de gran ayuda para arrojar luz sobre numerosos temas de investigación respecto al objeto sobre el que trata este artículo. Se trata de "Prensa del Movimiento de Madrid" y "Lucio del Álamo".

Por otro lado, no hay que olvidar que a través de estos fondos se pueden estudiar, por sus relaciones, otros medios de comunicación de carácter privado, desaparecidos o no, como pueden ser La Gaceta del Norte, El Alcázar, Olimpia..., y no solo prensa de Madrid, sino de toda España.

A nivel de organización de fondos en el Archivo Regional de la Comunidad de Madrid, se han incluido ambos como integrantes del grupo de fondos Movimiento Nacional, ya que se vinculan, por razón de sus funciones o actividades, a entidades pertenecientes al llamado Movimiento Nacional, como término que integra el conjunto de organismos y personas que formaron parte de la estructura política e institucional del régimen franquista.

\section{Desarrollo}

En las siguientes páginas figura una introducción sobre cada fondo, su origen, su organización y sus series más destacadas para la investigación de la prensa en el periodo citado. 
Además, se acompañan los cuadros de clasificación de cada fondo, con los tipos y órganos que lo componen, las fechas extremas y el número de registros descriptivos asociados. Asimismo, como anexo se incluye una relación de los diversos medios que podemos encontrar en estos conjuntos documentales y que se han recogido como puntos de entrada por índices onomásticos.

\subsection{Fondo "Prensa del Movimiento de Madrid"}

El fondo "Prensa del Movimiento de Madrid" custodiado en el Archivo Regional contiene 27,48 metros lineales ${ }^{2}$ de documentación relativa a la gestión y administración de tres de los medios de prensa editados en la provincia de Madrid entre 1940 y 1977, el diario Arriba, el diario Marca y el semanario 7 Fechas.

Las fechas extremas son 1927-1976 aunque la mayor parte del fondo se corresponde con los años 50 y 60 del pasado siglo.

Prensa del Movimiento de Madrid se sitúa en la calle Larra no 14 de la capital, en un edificio modernista que había sido construido en 1908 para acoger las máquinas y la redacción del semanario ilustrado Nuevo Mundo, hasta 1917, y posteriormente a las cabeceras y talleres de La Voz y El Sol.

En mayo de 1937 se produce la incautación del inmueble por comités obreros, pasando El Sol a convertirse durante más de un año en órgano de comunicación del Partido Comunista de España (Mateos Fernández, 2002: 623). Posteriormente, en marzo de 1939, y coincidiendo con la entrada de las tropas franquistas en Madrid, la redacción del diario $E / \mathrm{Sol}$ fue confiscada por un grupo de falangistas, pasando a imprimirse en sus talleres, desde ese mismo día, la edición del antiguo diario Arriba.

Se imprimen en este edificio las siguientes cabeceras:

- Diario Arriba, órgano oficial de prensa de Falange Española. Fundado como semanario por José Antonio Primo de Rivera el 21 de marzo de 1935, un año más tarde fue suspendido por orden del gobierno de la II República.

El 29 de marzo de 1939 comenzó a editarse como diario hasta el 16 de junio de 1979 en que se publica su último número.

- Diario Marca, fundado el 21 de diciembre de 1938 en San Sebastián por Manuel Fernández Cuesta Merelo, con carácter semanal y bajo el subtítulo de "Semanario Gráfico de los Deportes". En 1940 traslada su sede a Madrid, con domicilio en la calle Marqués de Cubas, hasta 1955 en que traslada su sede a la calle Larra no 14. Desde 1942 adquiere carácter diario, constituyendo la publicación que mayor

2229 cajas de archivo y un cajón planero descritos en 550 registros descriptivos 
tirada y número de lectores tenía de toda la entidad hasta la aparición del diario AS (1973). En 1984 fue puesto a la venta y adquirido por el Grupo Recoletos.

- Semanario 7 Fechas, fundado por Lucio del Álamo Urrutia en 1949, se configura como revista semanal (salía los martes), aunque con formato de periódico, en la que se integraba un resumen acompañado de numerosas fotografías de los temas que habían sido actualidad durante la semana. Desde los años 60 el semanario pierde popularidad y en 1977 se produce su cierre definitivo.

- Revista El Ruedo, fundada por Manuel Fernández-Cuesta en 1944 como suplemento taurino del diario Marca. El éxito de la publicación lo convierte desde el 21 de noviembre de 1946 en revista especializada que se edita con el subtítulo de "Semanario gráfico de los toros". El último ejemplar editado es de 1 de febrero de 1977, pero desgraciadamente no se conservan documentos en el Archivo Regional de esta publicación.

En 1963 se produce el traslado de la dirección, redacción y administración a un moderno edificio, obra del arquitecto Francisco de Asís Cabrero, situado en la entonces denominada Avenida del Generalísimo no 142 (hoy Paseo de la Castellana no 272, en el que se encuentra la Dirección General del Catastro) y allí permanecerá hasta su disolución.

La influencia de la Prensa del Movimiento a mediados de la década de 1950 era enorme: en 1956, de los 104 diarios publicados en España, 38 pertenecían al Movimiento, lo que suponía que un $32 \%$ de la prensa española estaba en manos del partido único. Sin embargo, conforme pasó el tiempo su influencia disminuyó: si en 1945 el 41\% de la prensa española estaba controlada por FET y de las JONS, para 1970 sólo controlaba un 26\% (Reig y Langa, 2011: 147).

La muerte de Francisco Franco y el desmantelamiento del aparato burocrático y político del régimen franquista, desemboca en la supresión de Prensa del Movimiento.

En 1977, por Decreto Ley 708/1977, de 15 de abril, se establece el cambio de nombre y estructura del grupo mediático, pasando a constituirse en organismo autónomo dependiente primero del Ministerio de Información y Turismo y posteriormente del Ministerio de Cultura, con la denominación de Medios de Comunicación Social del Estado.

En 1982, por la Ley 11/1982, de 13 de abril, se autoriza a Medios de Comunicación Social del Estado, a enajenar, mediante subasta pública, cada uno de los medios de prensa integrados en el citado organismo, procediéndose a la liquidación definitiva de aquellos que no encuentren adquiriente en dicho proceso.

En 1984 el Ministerio de Cultura determina en unas instrucciones internas que ante el proceso de subasta o cierre de periódicos pertenecientes al extinguido Medios de Comunicación Social del Estado, es necesario tomar medidas para que los archivos de 
los referidos medios (archivos administrativos, archivos de material de prensa, guiones, y archivo fotográfico) tengan en el futuro la utilidad que, desde el punto de vista de la información pública y la investigación, sea más idónea (Naseiro Ramudo, 2013: 28)

Para ello dispone que:

- En los casos en que se determine la liquidación y cierre de los periódicos sin continuidad en sus funciones, los archivos de los medios de prensa se pondrán a disposición del Archivo Histórico Provincial que corresponda.

- En los casos en que los nuevos titulares pretendan la continuación de su funcionamiento, se deberán incluir como bienes objeto de transacción aquellos materiales de sus archivos que sean necesarios para ello. Mientras que toda aquella documentación administrativa que no sea necesaria para la continuidad de las actividades de los medios se pondrá a disposición del citado Archivo Histórico Provincial.

La falta de Archivo Histórico Provincial en Madrid explica la presencia de fondos producidos por las entidades públicas antecesoras a la administración autonómica en el Archivo General de la Administración.

En el caso de la Prensa del Movimiento de Madrid solo el diario deportivo Marca pasó a manos de un grupo empresarial privado, mientras que la caída de lectores había precipitado anteriormente el cierre de 7 Fechas y El Ruedo en 1977, y de Arriba en 1979.

Esta información contradice el hecho de que el Archivo Regional posea, como venimos diciendo, fondos producidos por Prensa del Movimiento de Madrid. La realidad es que, después de una investigación sobre su historia archivística, estos documentos se encontraron en 1986 en el edificio que había sido sede de esta entidad, situado en la calle Larra no 14 de Madrid.

El ingreso viene motivado por la adquisición por parte de la Comunidad de Madrid, en ese mismo año, del citado edificio, en un principio con objeto de adecuarlo para instalar el futuro Archivo Regional, aunque finalmente el proyecto no salió adelante ${ }^{3}$.

Entre los días 1 y 2 de julio de 1986 se empaqueta la documentación y se traslada a la sede del entonces denominado Centro Regional de Archivos situado en la calle Talavera no 11 de Madrid, donde se procedió a su fumigación.

Posteriormente, el fondo se traslada al depósito del Archivo Central de la Consejería de Cultura, y dada la falta de espacio, el fondo queda depositado, de nuevo, en las dependencias de la calle Talavera no 11, pasando en 1995 a la nueva sede del Archivo Regional situada en la calle Amaniel. Es en 2003 cuando se trasladan la totalidad de los fondos a la nueva sede del Archivo Regional ubicada en el Complejo El Águila (antigua 
fábrica de cervezas El Águila), situada en la calle Ramírez de Prado no 3 de Madrid, lugar en el que se custodian en el momento actual.

Entre 2019 y 2020 se procede en la Unidad de Descripción del Archivo Regional de la Comunidad de Madrid a la revisión y actualización de los instrumentos descriptivos de este fondo con la finalidad de actualizar y proporcionar una mayor información a los potenciales usuarios del mismo.

La organización dada al fondo se asienta en cuatro agrupaciones: Administración, Talleres, Dirección de Medios y Otros Fondos. A su vez Administración se subdivide en Asuntos Generales, Contabilidad, Personal, Circulación, Publicidad y Almacén. Dirección de Medios se subdivide a su vez en Dirección del diario Arriba, Dirección del diario Marca y Dirección del semanario 7 Fechas.

Se incluye el cuadro de clasificación del fondo "Prensa del Movimiento de Madrid" en el que se puede comprender la organización funcional dada al mismo por el equipo de descripción del fondo, los tipos documentales reflejo de esas funciones, y su alcance temporal.

Las series más voluminosas son las relacionadas con las principales fuentes de ingresos de la entidad: publicidad inserta (Publicidad) y distribución de ejemplares (Circulación). Suponemos que estas series, en gran medida parciales, tienen su continuidad en el Archivo General de la Administración, al menos no es aventurado pensarlo.

Señalamos los datos más significativos de cada una de ellas. Bajo el epígrafe Administración/Publicidad encontramos:

1. Órdenes de inserción de anuncios. Serie compuesta por 115 registros descriptivos fechados entre 1951 y 1965, de la cual el documento principal es un impreso denominado orden de publicidad -en un principio más diverso y que se normaliza con el tiempo-, enviado al departamento de publicidad por el agente y/o agencia de publicidad en el que se determinan las características del anuncio que se va a insertar, se fija el precio que deberá pagar el anunciante, y se registra el medio de prensa y fecha en que aparecerá el anuncio.

Los datos que figuran habitualmente en este impreso son:

- Nombre del agente o de la agencia.

- Texto del anuncio.

- Tamaño y forma (alto, ancho, columnas o número de modelo de anuncio).

- Precio a tarifar.

- Fecha y periódico en el que debe insertarse el anuncio.

- En algunos casos aparecen imágenes, dibujos a lápiz o montajes. 
Nuevas fuentes documentales para el estudio de la prensa en España durante el franquismo: el archivo regional de la Comunidad de Madrid

María del Pilar Solís Herráiz

2. Registro de órdenes de publicidad. Serie compuesta por 32 registros fechados entre 1956 y 1963 . Formada por un impreso normalizado empleado por el departamento de publicidad en el que se apuntan diariamente y por cada periódico adscrito los datos de las Órdenes de inserción de anuncios mencionadas anteriormente.

El impreso tiene formato de registro en el que aparecen los siguientes datos:

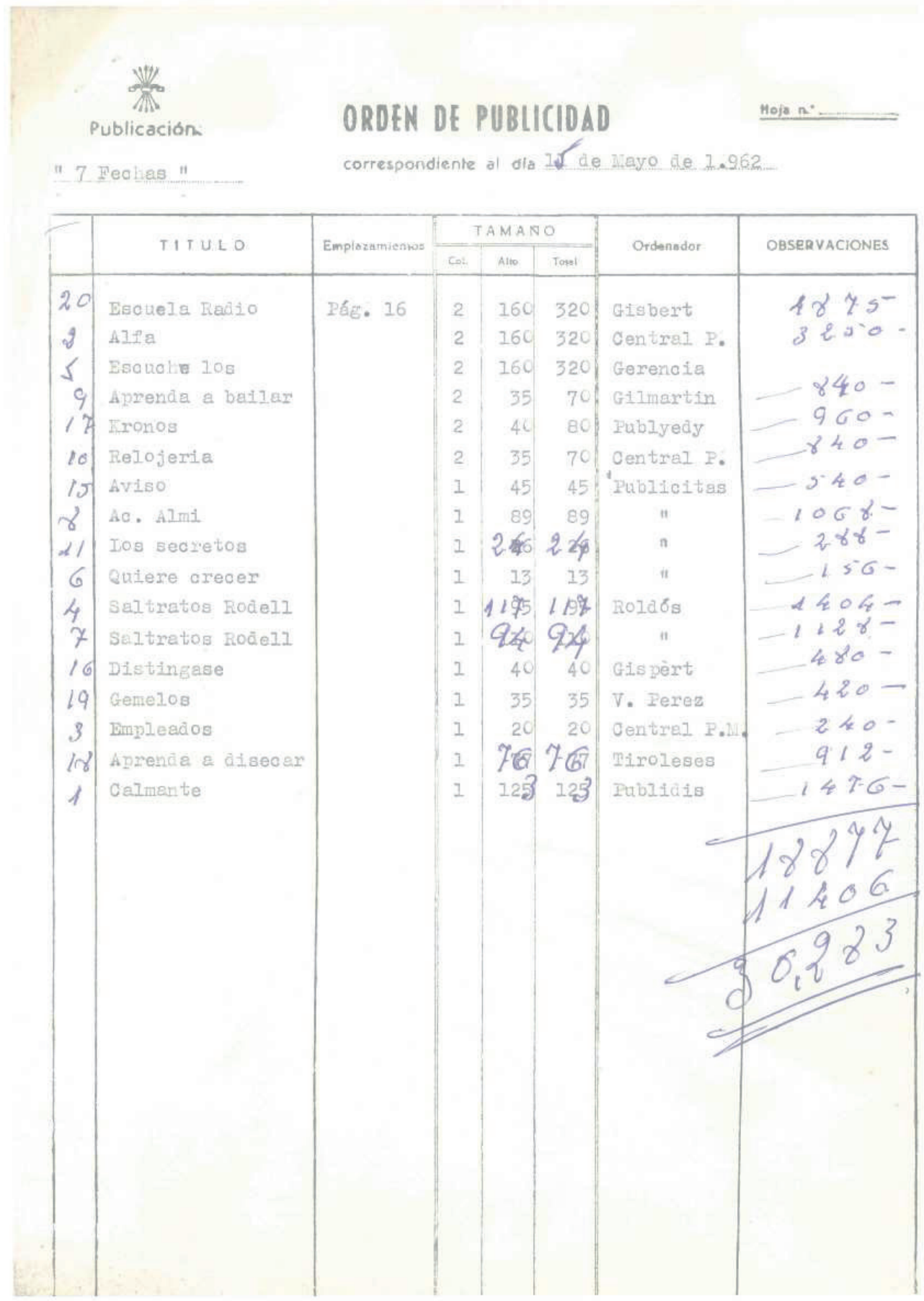

Fig. 1. ARCM. Fondo "Prensa del Movimiento de Madrid" Sign. 12994/1. Ejemplo del tipo documental Registro de Órdenes de publicidad. 
En la cabecera: nombre del periódico y fecha completa de las Órdenes de inserción de anuncios que se van a registrar. En las columnas figuran:

- Título.

- Emplazamiento.

- Tamaño (Columna, alto, total).

- Ordenador (agente publicitario que ordena la inserción).

- Observaciones: contiene anotaciones manuscritas en cifras posiblemente correspondientes al precio del anuncio, y al sumatorio del total de ingresos de ese día en concepto de publicidad.

- En algunos casos se incluyen también los documentos justificativos de la inserción de anuncios en el día.

Ambos tipos documentales aportan una valiosa información, entre otros ámbitos, para estudios sobre la publicidad, ingresos, principales empresas, evolución de las mismas entre otros, que de otra forma sería imposible realizar.

Bajo el epígrafe Administración/ Circulación encontramos:

3. Registro auxiliar de entrada y salida de documentos. Compuesta por un total de 52 registros fechados entre 1950 y 1963. Aparece dividida en dos subseries, en función de si el registro corresponde a los documentos remitidos y/o enviados por los corresponsales (responsables de los puntos de venta, distribuidos por las ciudades y pueblos de España) o por los suscriptores (responsables del control de los suscriptores).

Respecto a los Corresponsales, está formada por los documentos emitidos por los puntos de venta y por la administración de la Prensa del Movimiento de Madrid para la gestión de la distribución de los ejemplares de Arriba, Marca y 7 Fechas. Contiene entre otros documentos papeletas de pedidos, modificaciones de pedidos, condiciones de la corresponsalía, letras de cambio, facturas, incidencias, consultas de los vendedores de prensa de diferentes ciudades en toda la geografía española.

Respecto a Suscriptores está formada tanto por solicitudes de alta como suscriptores, como modificación o bajas de la suscripción por particulares o entidades públicas o privadas, remitidas desde diferentes puntos tanto nacionales como europeos y otras partes del mundo 4 .

Es por lo tanto una fuente de información muy interesante para conocer la implantación de los distintos medios falangistas en cada provincia española y en otros países, la preeminencia de las publicaciones generales frente a las locales o la evolución de los distintos medios a lo largo de más de una década.

4 Véase signatura 12960/1 
Nuevas fuentes documentales para el estudio de la prensa en España durante el franquismo: el archivo regional de la Comunidad de Madrid

Otros tipos documentales interesantes, aunque poco numerosos y más parciales que los señalados anteriormente, son los Partes de suscripción y el Registro diario del movimiento de altas y bajas de suscripciones al diario Arriba, al diario Marca y al semanario 7 Fechas. Constituyen un resumen de la evolución de las suscripciones de forma diaria.

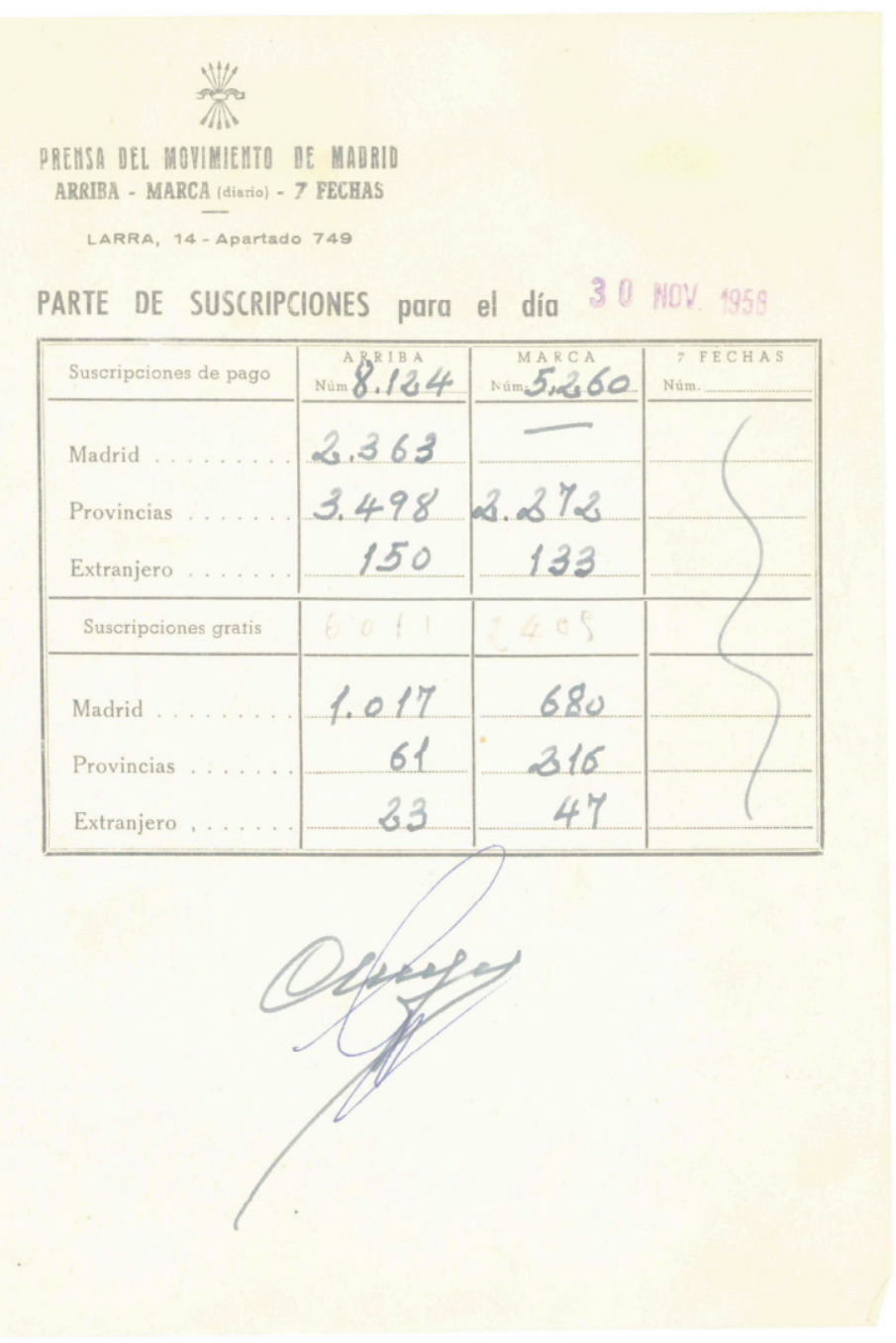

Fig. 2. ARCM. Fondo "Prensa del Movimiento de Madrid" Sign. 13042/3. Ejemplo del tipo documental Partes de suscripción.

Otro tipo documental que puede ser interesante como fuente de investigación es el Registro auxiliar de entrada y salida de documentos, formado por la correspondencia mantenida por el gerente del diario Arriba con diferentes ámbitos del diario: el administrador, el director, el redactor jefe..., relativa a la designación de redactores para cubrir noticias, la aprobación de viajes, las altas y bajas de la plantilla, las incidencias con la censura, comentarios y directrices, propuestas. 


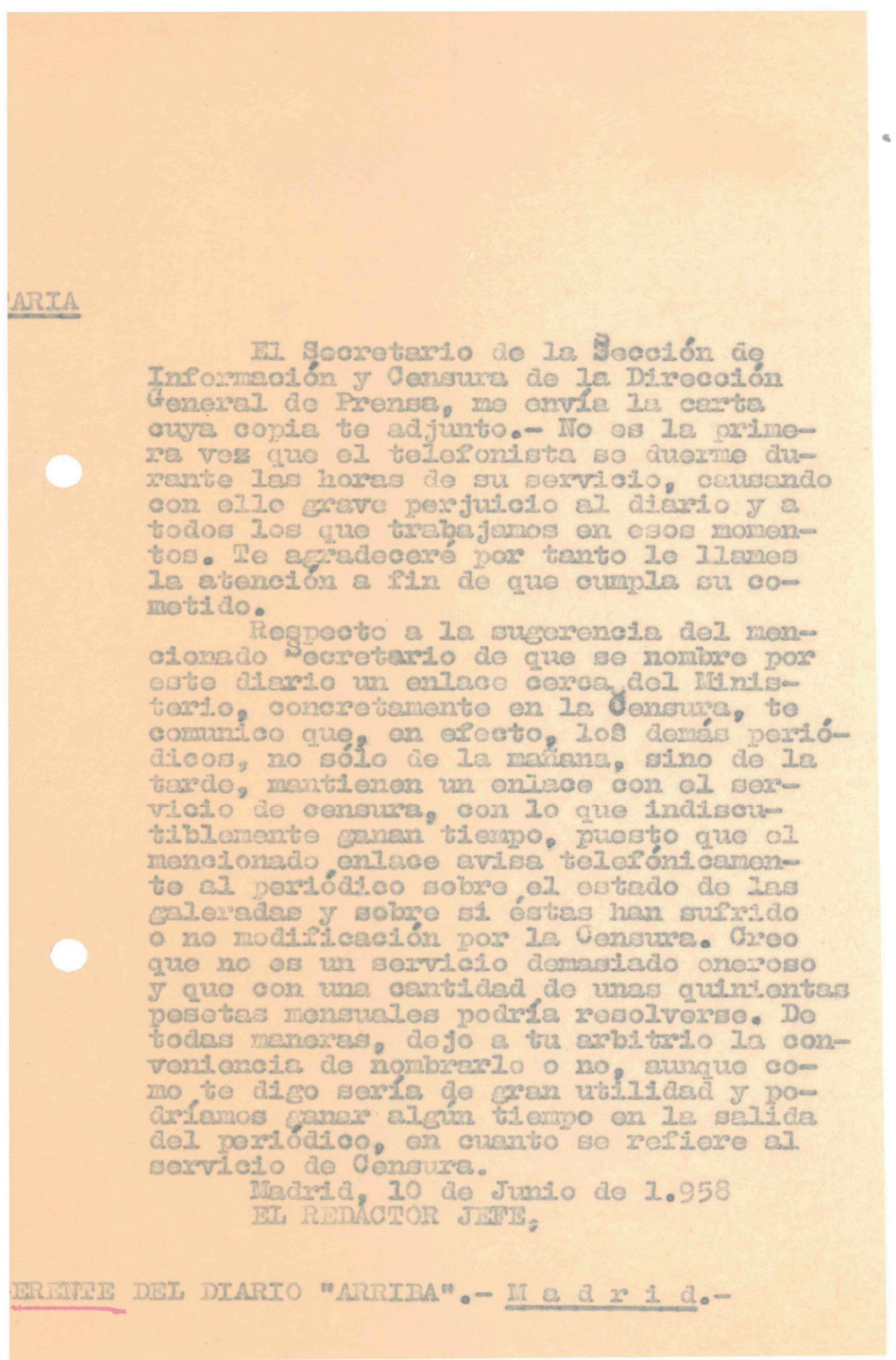

Fig. 3. ARCM. Fondo Prensa del Movimiento Sign. 13045/4. Ejemplo del tipo documental Registro auxiliar de entrada y salida de documentos.

Desde el punto de vista de su contenido, señalar la importancia de la serie denominada Correspondencia adscrita al apartado "Dirección de medios", formada por un total de 249 registros fechados entre los años 1942 y 1958. Se trata de los únicos documentos de este fondo referentes a los órganos de dirección de los diarios y revistas adscritos a 
Prensa del Movimiento de Madrid, siendo mayoritarias las cartas dirigidas a Rafael García Serrano, y en menor medida a Vicente Cebrián Carabias y Manuel Vázquez Prada, directores y subdirectores del diario Arriba, a lo largo del año 1957.

Destaca la correspondencia mantenida con otros directores de diarios como Francisco Villagordo Montalbán, director del diario Amanecer o R. Carreño García-Tudela, administrador del diario Los Sitios de Gerona.

También aparecen como emisores de las misivas políticos y directores de otros medios de comunicación, personas de instituciones públicas y privadas, o particulares, siendo las más interesantes aquellas mantenidas con colaboradores como Ernesto Giménez Caballero, Miguel Delibes o Gonzalo Torrente Ballester, y con la Dirección General de Prensa por sus datos sobre censura y gestión de la información.

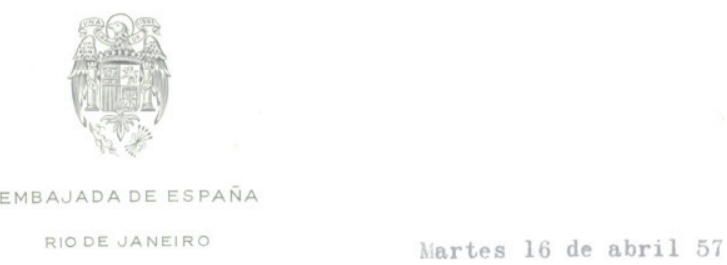

Veo que no os acordais ni de escribiame ni de publicar lo que os mando.? Salieron mis dos ultımos articulos?Yo no loo he visto en los ejemplares que aqui llegan.0s envio este con fotos sobre Brasil. Sino los quereis publicar decidmelo.Y escribire en otro sitio que espero no haya de falta Sino
rmine
rme.

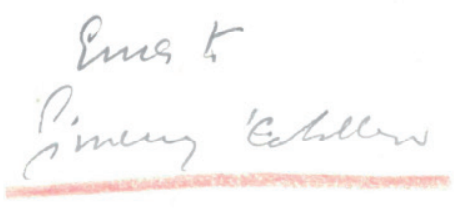

Fig. 4. ARCM. Fondo Prensa del Movimiento Sign. 13044/93. Carta de Ernesto Giménez Caballero al director del diario "Arriba" quejándose de la falta de publicación de sus artículos.

Es interesante señalar la presencia, en las series adscritas a Contabilidad, de datos de otras publicaciones ajenas al ámbito competencial y territorial del productor del fondo, pero que se confeccionaban en los talleres de la calle Larra no 14 como las revistas 
Olimpia (semanario deportivo catalán, suplemento de Solidaridad Nacional), Juventud (editada por el Frente de Juventudes) o Servicio (revista de temas educativos).

Además, el fondo incluye otras series documentales -reflejo de la gestión contable y de personal, del funcionamiento de los almacenes y de los talleres de edición-, todas ellas de gran interés, pero caracterizadas por su escaso volumen y fragmentación.

Tabla 1. Cuadro de clasificación de fondo "Prensa del Movimiento de Madrid".

\begin{tabular}{|c|c|c|}
\hline ÓRGANOS PRODUCTORES & $\begin{array}{l}\text { Fechas } \\
\text { Extremas }\end{array}$ & $\begin{array}{l}\text { № } \\
\text { registros }\end{array}$ \\
\hline \multicolumn{3}{|l|}{ ADMINISTRACIÓN } \\
\hline \multicolumn{3}{|l|}{ 1.1 ASUNTOS GENERALES } \\
\hline Contratos & $(1949-1953)$ & 3 \\
\hline Expedientes de obras menores & $(1957-1958)$ & 1 \\
\hline Expedientes de suministros & $(1948-1958)$ & 13 \\
\hline Expedientes de pago & $(1953-1955)$ & 5 \\
\hline Registro auxiliar de entrada y salida de documentos & $(1951-1959)$ & 6 \\
\hline Recibos & (1957) & 2 \\
\hline Relaciones & $(1955)$ & 1 \\
\hline Resúmenes & $(1954)$ & 2 \\
\hline \multicolumn{3}{|l|}{ 1.2 CONTABILIDAD } \\
\hline Facturas & $(1952-1962)$ & 7 \\
\hline Justificantes de operaciones bancarias & $(1956-1960)$ & 7 \\
\hline Partes diarios de ingresos y gastos & $(1955-1960)$ & 3 \\
\hline Registro auxiliar de entrada y salida de documentos & $(1951-1961)$ & 7 \\
\hline Registro diario de gastos por colaboraciones & $(1955-1957)$ & 1 \\
\hline Relaciones & (1956 aprox) & 1 \\
\hline \multicolumn{3}{|l|}{ 1.2.1 Caja } \\
\hline Letras de cambio & $(1959-1961)$ & 3 \\
\hline Recibos de pago & $(1959-1962)$ & 1 \\
\hline \multicolumn{3}{|l|}{ 1.3 PERSONAL } \\
\hline Declaraciones juradas & $(1952)$ & 1 \\
\hline Recibos de nóminas & (1959) & 1 \\
\hline Registro de nóminas & $(1956-1958)$ & 1 \\
\hline Relaciones & $(1951-1954)$ & 2 \\
\hline Resúmenes & (1955) & 1 \\
\hline
\end{tabular}




\subsection{CIRCULACIÓN}

\subsubsection{Suscriptores}

Partes de suscripciones

(1958)

Registro auxiliar de entrada y salida de documentos

(1950-1963) 24

Registro diario de altas y bajas

(1967-1976) 3

\subsubsection{Corresponsales}

Registro auxiliar de entrada y salida de documentos

$(1951-1962) \quad 28$

\subsubsection{Reparto}

Notas

(1972-1976) 2

Partes de transporte

1

Registro diario de ejemplares para el reparto

(1963-1975) 4

\subsection{PUBLICIDAD}

Órdenes de inserción de anuncios

(1951-1965) 115

Registro de órdenes de publicidad

\subsection{ALMACÉN}

Partes de venta de residuos

$(1958 ; 1961) \quad 2$

Registro diario de salida de materiales

(1957-1961) 2

Vales de entrega de suministros

(1948-1961) 6

\section{TALLERES}

Partes diarios de trabajo

Registro de bobinas de papel

(1955-1962) 5

(1952-1954) 1

\section{DIRECCIÓN DE MEDIOS}

\subsection{Dirección del diario ARRIBA}

Certificados

(1957)

Correspondencia

\subsection{Dirección del diario MARCA}

Correspondencia

\subsection{Dirección del semanario 7 FECHAS}

Correspondencia

\section{OTROS FONDOS}

\subsection{DIARIO EL SOL}

Correspondencia

Dosieres de prensa

(1927-1933) 1


Informes

Justificantes de gastos

\subsection{OTROS}

Dibujos
(1960-67

1

aprox.)

\section{Fondo "Lucio del Álamo"}

El fondo "Lucio del Álamo" custodiado en el Archivo Regional está formado por los documentos producidos a lo largo de su vida, tanto a nivel profesional como escritor y periodista, como a nivel político como delegado provincial de Educación Popular de Vizcaya, delegado nacional de Prensa y Propaganda y director de Radio Nacional de España.

Estos documentos son adquiridos por la Comunidad de Madrid en el año 2006 ya que uno de sus objetivos según la Ley 4/1993, de 21 de abril, de Archivos y Patrimonio Documental de la Comunidad de Madrid es el acrecentamiento del patrimonio documental que pueda ser de interés para el conjunto de los ciudadanos madrileños. Para ello, cuenta con las figuras legales del depósito, de la donación o de la compra de documentos, fondos documentales o colecciones.

Este fondo custodiado en el Archivo Regional ocupa 1,92 metros lineales ${ }^{5}$. Las fechas extremas son 1928-1967.

Podemos encontrar referencias a la biografía de Lucio del Álamo en diversas fuentes: la Auñamendi Eusko Entziklopedia, la Asociación de la Prensa de Madrid... pero son bastante parciales. Esperamos que la difusión de este fondo documental sirva también para dar a conocer en toda su amplitud su trayectoria profesional.

Lucio del Álamo Urrutia nace en Saratxo (Amurrio, Álava) el 2 de marzo de 1913, hijo de Nicasio del Álamo y Tomasa Urrutia. Se casa en 1942 con Juanita Gómez, cursa estudios de Derecho y de Filosofía y Letras en la universidad de Deusto, hasta el momento en que el gobierno de la II República cierra esta institución por su pertenencia a la Compañía de Jesús. Continúa entonces la carrera en Valladolid y en Madrid.

Se forma como periodista en la Escuela de Periodismo creada por el diario El Debate, considerado el primer centro de formación para periodistas en España, fundada por Ángel Herrera Oria en Madrid, y que funciona como tal entre los años 1926 y 1936, con

51 cajón de planero y 16 unidades de instalación normalizadas descritas en 256 registros descriptivos. 
un programa de estudios inspirado en la universidad de Columbia (Nueva York) y en la práctica del oficio de los diarios The Globe, The World y The New York Times.

En 1934 ingresa como redactor en el diario La Gaceta del Norte, editado en Bilbao, medio al que permanecerá vinculado hasta 1942, compaginando el trabajo como corresponsal y colaborador del periódico con otros puestos en diferentes medios de prensa y en cargos de responsabilidad pública ${ }^{6}$. Militante de las JONS, al fusionarse éstas con Falange Española pasó a formar parte de la nueva organización.

Durante la Guerra Civil figura como miembro del Batallón de Montaña Flandes no $5^{7}$ enviado a los frentes de Asturias y Zaragoza, si bien fue declarado parcialmente inútil debido a su bronquitis crónica, regresando en febrero de 1939 a su anterior puesto en La Gaceta del Norte como redactor militarizado.

Finalizada la guerra, reside en Bilbao, donde es nombrado director de la Hoja del Lunes (1940), designado concejal del Ayuntamiento de Bilbao (1941-1942), diputado por la provincia de Vizcaya, y delegado provincial de Educación Popular (mayo-noviembre de 1943).

En 1944 se traslada a Madrid, donde pasa a ocupar diversos puestos vinculados al Movimiento Nacional: director de Radio Nacional de España (1943-1958), Jefe Nacional de Radiodifusión (1944) y delegado nacional de Prensa del Movimiento (1946-1953)

Como periodista cabe señalar que fue director del diario Marca (1947-1953), El Alcázar (1968-1971) y Hoja del Lunes de Madrid (1973-1977), así como fundador y director del semanario 7 Fechas (1949-1977).

Ejerce desde los años 50 una actividad asociativa relacionada con la profesión periodística: presidente de la Asociación de la Prensa de Madrid entre los años 1951 y 1955, y posteriormente entre 1967 y 1979 promueve la construcción de la urbanización denominada "Ciudad de los Periodistas" en el barrio de Peñagrande de Madrid; presidente del Sindicato Provincial de Prensa, Radio, Televisión y Publicidad, creado en 1965; y presidente de la Federación Nacional de Asociaciones de la Prensa de España y de la Mutualidad Laboral de periodistas.

Durante su carrera como periodista recibe numerosos premios y honores, entre los que destacan el Premio Nacional de Periodismo Francisco Franco (1964) por sus artículos publicados en Arriba, Hierro de Bilbao, La Actualidad Española y la Hoja del Lunes de Madrid, Gran Cruz de la Orden de Cisneros (1969), premio Mariano de Cavia (1970),

6 Signatura ARCM 73942/26

7 Signatura ARCM 73942/21

8 Signaturas ARCM 73948/21-3 
Gran Cruz de la Orden Civil de Alfonso X El Sabio (1971) y el premio Periodista de Honor (1976).

Fue autor además de varias obras literarias como Charlas del sábado (Prosas de guerra, de amor y de esperanza) (1941), El último muerto de la guerra de España (1944), Meditaciones sobre la independencia económica de España (1950), El muerto solo (1954) y Veinticinco años de paz vistos por 25 escritores españoles (1964).

Falleció en Madrid el 27 de junio de 1988.

El fondo se organiza siguiendo un criterio orgánico-funcional (véase el Cuadro de Clasificación del fondo), según los ámbitos de actividad desarrollados por el productor, lo que ha dado lugar a la formación de cuatro agrupaciones documentales.

- Actividad privada. Forman parte de este epígrafe los escasos documentos que se custodian relacionados con asuntos personales y particulares de Lucio del Álamo.

- Actividad profesional. Agrupa los documentos producidos o reunidos en el ejercicio de su carrera como periodista y escritor, que no se vinculan con el desempeño de cargos políticos o administrativos, por ejemplo, los relativos al proceso de redacción y publicación de El último muerto de la guerra de España, libro sobre la figura de Alfonso Churruca y Zubiría o la correspondencia entre Lucio del Álamo y Antonio González Martínez de Olaguibel (1901-1979), director y miembro del consejo de administración del diario La Gaceta del Norte, y con el que desarrolla una gran amistad forjada a partir de su trabajo como redactor en el periódico. Así, en cualquiera de las cartas que se custodian, se puede encontrar el tratamiento de un asunto familiar junto a un tema profesional y político, como la problemática de la escasez y provisión del papel para el periódico en la España de posguerra.

- Actividad política. Agrupa los documentos relacionados con el ejercicio de cargos públicos, entre los que destacan, por constituir en torno al $90 \%$ del fondo, los producidos y reunidos en su cargo como delegado nacional de Prensa y Propaganda. Se han clasificado siguiendo una estructura funcional, que obedece a las diferentes áreas de gestión de la entidad: Dirección, Administración, Contabilidad, Inspección, Colaboraciones, Personal, Publicidad.

- Actividad asociativa. Reúne un escaso volumen de documentos producidos por Lucio del Álamo en el ejercicio de la presidencia de la Asociación de la Prensa de Madrid y la Federación Nacional de Asociaciones de la Prensa de España, ya que en su inmensa mayoría se encuentran en el archivo de la Asociación de la Prensa de Madrid.

Las series de mayor volumen se relacionan, como hemos señalado, con su cargo de delegado nacional de Prensa y Propaganda. Este organismo, creado en 1937 y dependiente de FET y de las JONS, se encuadra tras la Guerra Civil en el aparato 
administrativo del régimen, con diferentes dependencias orgánicas a lo largo de su historia, pero en todos los casos con las funciones de control y gestión de los medios de comunicación adscritos a la llamada Prensa del Movimiento.

Los documentos custodiados en el Archivo Regional reflejan las relaciones entre estos diarios y el delegado nacional de Prensa, por lo que se convierten en fuente de información básica para el estudio y conocimiento de la prensa española durante el franquismo.

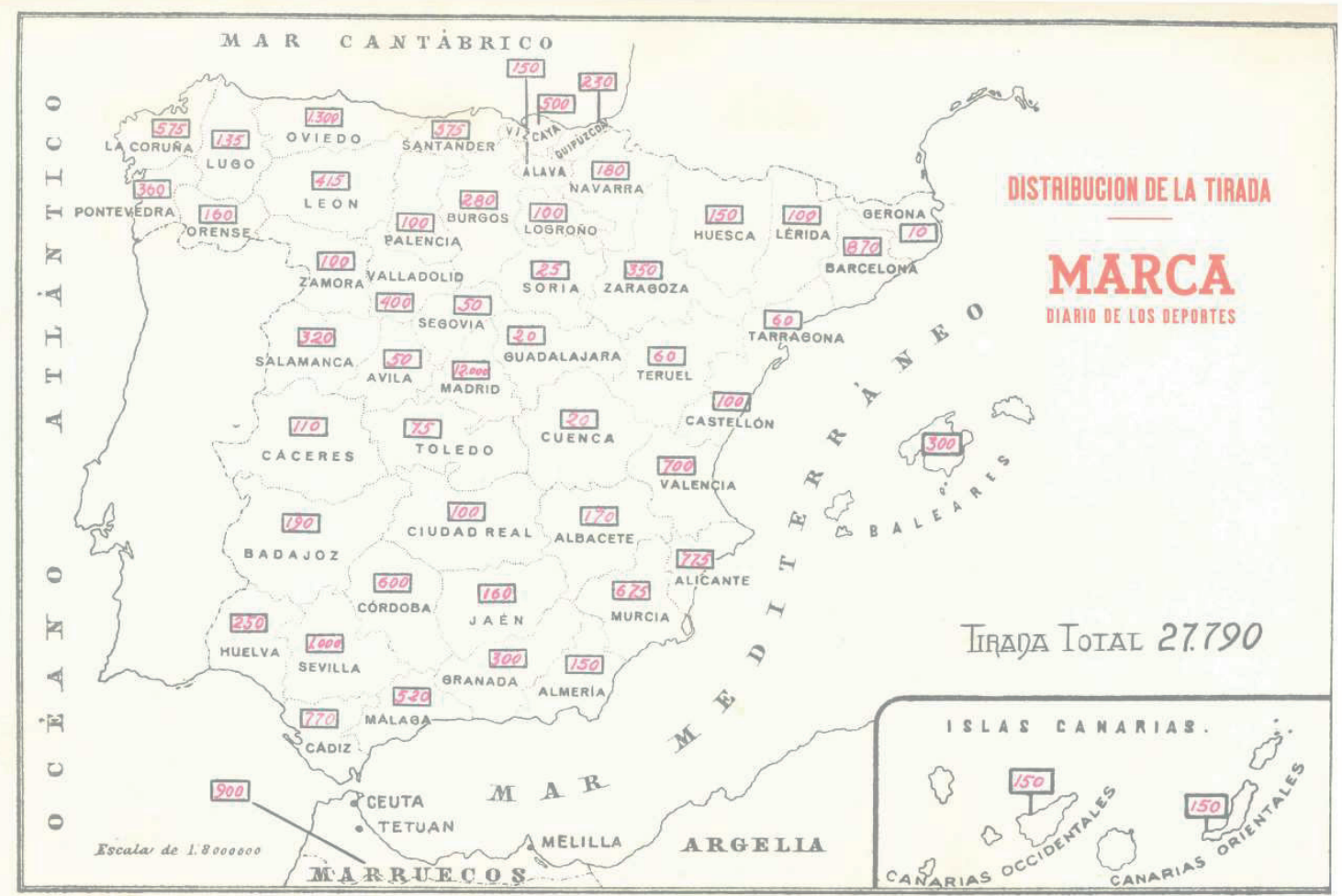

Fig.5. ARCM. Fondo "Lucio del Álamo" Sign. 73951/3. Mapa de España con la distribución por provincias de la tirada del diario Marca

Adscritas al área de Dirección, señalamos por el interés de su contenido y volumen, las siguientes series documentales:

- Dosieres: 18 registros fechados entre 1945 y 1952, que agrupan los documentos reunidos por el productor del fondo para su gestión sobre diferentes temas, como el reparto del cupo del papel o la regularización de las incautaciones de los medios de prensa, en casos como el del periódico El Sol, La Gaceta de Alicante, La Voz de Guipúzcoa o Prensa Gráfica y otros.

- Correspondencia: 12 registros, fechados entre 1945 y 1953, organizados de forma alfabética a partir de los apellidos del autor y/o destinatario de cada una de las cartas. Figuran las de personas de especial relevancia en el régimen franquista como Rafael Sánchez Mazas, José María Sánchez Silva, Ramón Serrano Suñer, 
Manuel Vázquez de Prada, Xabier de Echarri Gamundi, Pedro Laín Entralgo, Marjone Munde, Dionisio Ridruejo...

- Informes y notas: 16 registros, fechados entre 1946 y 1952, relativos a la situación general de la prensa en España o sobre determinados periódicos adscritos al Movimiento Nacional, que en ocasiones contienen información confidencial preparatoria de asuntos que eran tratados en gabinete entre Lucio del Álamo y sus superiores.

El área de Administración, relativa a la gerencia de los diferentes medios de prensa adscritos al grupo editorial, contiene la serie de mayor antigüedad y volumen del fondo: Dosieres. Está formada por 47 registros, fechados entre 1938 y 1953, en los que se agrupan los documentos relativos a la administración y gestión de un determinado medio. En líneas generales, encontramos correspondencia con la dirección y administradores de los periódicos, documentos referidos a plantillas y sueldos, presupuestos, gastos, ingresos, proveedores, beneficios obtenidos por las tiradas y la publicidad, asuntos de interés local y polémicas puntuales con otros medios.

Los medios de prensa y lugar de edición que tienen un registro propio en esta serie son los siguientes:

Tabla 2. Medios de prensa y lugar de edición con registro propio.

\begin{tabular}{ll} 
Medio de prensa & Lugar de edición \\
7 Fechas & Madrid \\
Alerta & Santander \\
Amanecer & Zaragoza \\
Arriba & Madrid \\
Arriba España & Pamplona \\
Ayer & Jerez de la Frontera \\
Baleares & Palma de Mallorca \\
Córdoba & Córdoba \\
El Español & Tarragona \\
El Pueblo Gallego & Vigo \\
F.E. & Sevilla \\
Falange & Las Palmas de Gran Canaria \\
Fotos & Madrid \\
Gol & Madrid \\
Hierro & Bilbao \\
Imperio & Zamora \\
\hline
\end{tabular}




\begin{tabular}{ll}
\hline Información & Alicante \\
Jaén & Jaén \\
Jornada & Valencia \\
La Mañana & Lérida \\
La Prensa & Barcelona \\
La Tarde & Madrid \\
La Tarde & Málaga \\
La Voz de Castilla & Burgos \\
La Voz de España & San Sebastián \\
La Voz del Sur & Cádiz \\
Levante & Valencia \\
Libertad & Valladolid \\
Línea & Murcia \\
Los Sitios & Gerona \\
Maravillas & Madrid \\
Marca & Madrid \\
Mediterráneo & Castellón de la Plana \\
Nueva España & Huesca \\
Odiel & Huelva \\
Patria & Granada \\
Proa & León \\
Sur & Málaga \\
\hline Yugo & Almería \\
\hline
\end{tabular}

Del área de Contabilidad señalar los Informes económicos relativos a ingresos y gastos de los diarios, a través de los cuales es posible el estudio de la viabilidad económica de este grupo editorial.

Por otro lado, podemos destacar que el área de Inspección contiene unas series interesantísimas por su contenido ya que son reflejo de las funciones de control ejercidas por Lucio del Álamo como delegado nacional de Prensa, Expedientes de inspección de servicios e Informes de inspección desde el año 1947 a 1951.

Los Informes de inspección de casi veinte medios de comunicación del grupo vienen acompañados en muchos casos por las hojas de inspección, correspondencia y un informe al respecto. Se examinan durante un mes los números editados y se realizan 
observaciones a diferentes temas. Tratan asuntos muy variados, desde la marcha económica del diario en cuestión, los artículos de carácter político, la calidad técnica de los mismos, la distribución de los cupos de papel, hasta el tratamiento de noticias locales, nacionales e internacionales, entre otros.

Por último, el único Expediente de inspección de servicios es referente al diario Unidad de San Sebastián por problemas de tipo laboral.

Adscritos al apartado de Secretarías se encuentran series como Correspondencia e Informes muy interesantes por ser documentos relacionados con Rodrigo Vivar Téllez, que ocupó puestos como gobernador civil de Vizcaya, vicesecretario y secretario general del Movimiento, cargo de suma importancia en el régimen. Estas secretarías son ejercidas de forma oficiosa por Lucio del Álamo ya que, hasta el momento, no hemos encontrado nombramiento oficial al respecto.

Se incluye el cuadro de clasificación del fondo "Lucio del Álamo" en el que se puede comprender la organización funcional dada al mismo por el equipo de descripción del fondo, los tipos documentales reflejo de esas funciones, y su alcance temporal.

Tabla 3. Cuadro de clasificación fondo "Lucio del Álamo"

ACTIVIDAD

Fechas Extremas № registros

\section{ACTIVIDAD PERSONAL}

Correspondencia

1944-1967

Dosieres 1928

Dosieres de prensa

1947-1951

Folletos

Invitaciones

1942-1950

Menús

Pasaportes

Revistas

\section{ACTIVIDAD PROFESIONAL}

Artículos

Contratos

1942-1949

Correspondencia

Expedientes de publicaciones 


\section{ACTIVIDAD POLÍTICA}

\subsection{Delegado Provincial de Educación Popular de Vizcaya}

Dosieres de prensa

1943

Nombramientos

1943

\subsection{Director de Radio Nacional de España}

Correspondencia

1943-1944

Dosieres de prensa

Informes

\subsection{Delegado Nacional de Prensa (1938-1953)}

\subsubsection{Dirección}

Artículos

1946-1952

12

Certificados

1948-1952

1

Correspondencia

1945-1953

12

Dosieres

1945-1952

18

Dosieres de prensa

1945-1950

4

Expedientes de información de servicios

1948-1951

Folletos

1946-1948

1

Fotografías

1943

1

Informes

1946-1952

15

Memorias

1950

Nombramientos

1945-1952

3

Notas

1950-1951

Planos

1946-1952

Proyectos

1946-1952

Relaciones

1946-1952

Revistas

1952

Solicitudes

\subsubsection{Administración}

Circulares

Correspondencia

\subsubsection{Contabilidad}

Cuentas 
Informes económicos

Saldos bancarios

\subsubsection{Inspección}

Estadísticas

1949-1950

Expedientes de inspección de servicios

Informes de inspección

\subsubsection{Colaboraciones}

Correspondencia

1947-1952

Presupuestos

\subsubsection{Personal}

Escalafones

1948

Expedientes de reclamación de cantidades

Proyectos

Relaciones

\subsubsection{Publicidad}

Estadísticas

1950

Informes

1947-1951

Normas de procedimiento

1951

1947-1950

1946-1952

Expedientes de reuniones

1945-1952

Tarifas de publicidad

1948-1949

1946-1952

\subsection{Consejero del Consejo Asesor de Prensa} 15

\subsection{Secretarías políticas}

\subsubsection{Secretaría gobernador civil de Vizcaya}

Correspondencia

1941

3.5.2 Secretaría de la Vicesecretaría General del Movimiento

Circulares

Correspondencia

Discursos

Dosieres

Expedientes de información de servicios

Expedientes de reclamación de cantidades

Informes

Ponencias
1946

1944-1947

1934-1944

1945

1946

1945-1946

1943-1949

1944-1945
1

1

1

2

1

1

4

1

4

1

1

1

1

1

1

6

1

1

1

2

12

1 


\section{ACTIVIDAD ASOCIATIVA}

\subsection{Presidencia de la Asociación de la Prensa de Madrid}

Correspondencia

1950-1953

Discursos

\subsection{Presidente de la Federación Nacional de Asociaciones de la Prensa de España}

\section{Conclusiones}

La organización llevada a cabo por la Unidad de Descripción del Archivo Regional de la Comunidad de Madrid de los dos fondos de los que hemos hablado queda expresada en los cuadros de clasificación que incorporamos para una mayor claridad y conocimiento de los mismos.

El resultado de los trabajos llevados a cabo se muestra en los instrumentos de descripción, inventarios (para su control y consulta) disponibles en el Archivo Regional de la Comunidad de Madrid.

Es interesante resaltar cómo dos fondos, con un origen tan diferente y con unas vicisitudes tan aparentemente poco archivísticas, puedan y deban ser, en el futuro, base para investigaciones sobre un periodo y una temática que parece que está siendo objeto de la mirada de la investigación actual. "Con el siglo XXI se ha desplegado una gran actividad investigadora sobre múltiples cabeceras provinciales, cuyas radiografías se han realizado, en muchos casos, en función de la disponibilidad y solidez de las fuentes consultadas" (Sanz Hernando, 2017: 27).

La disponibilidad y solidez de las fuentes aquí presentadas creemos que abre un amplio panorama para la investigación. Este es nuestro interés, dar a conocer a un elenco lo más amplio posible de investigadores, las posibilidades de estudio que existen en el Archivo Regional de la Comunidad de Madrid al respecto.

El acceso de los ciudadanos a los Archivos de la Comunidad de Madrid es libre y gratuito, previa presentación del Documento Nacional de Identidad, pasaporte o documento equivalente.

No se precisa de acreditación especial para la consulta de los documentos. Las limitaciones de cualquier consulta son las que establece la normativa vigente en materia 
de acceso y las que se derivan del estado de conservación de los documentos. Con el fin de garantizar la conservación del patrimonio documental original, si el documento que se desea consultar está reproducido, se sirve preferentemente la reproducción del mismo.

Se puede solicitar cita previa para acudir a las Sala de Consulta del Archivo Regional de la Comunidad de Madrid, pudiendo obtener reserva de mesa y facilitando la información suficiente para que, en la medida de lo posible, tengan preparados los documentos que desee consultar. Para ello, es necesario cumplimentar un formulario ${ }^{9}$.

\section{Referencias bibliográficas}

DE LAS HERAS PEDROSA, C. (2000): La prensa del movimiento y su gestión publicitaria (1936-1984), Málaga, Universidad de Málaga.

DE LAS HERAS PEDROSA, C. y JAMBRINO MALDONADO, C. (2014): "La publicidad en la prensa del movimiento". Historia y Comunicación Social, 19, pp. 847-860 https://doi.org/10.5209/rev_HICS.2014.v19.46563. [Accessed 6 July 2021].

MARTÍN DE LA GUARDIA, R. (1994): Información y propaganda en la Prensa del Movimiento. Libertad de Valladolid, 1931-1979, Valladolid, Universidad de Valladolid, Secretariado de Publicaciones.

MATEOS FERNÁNDEZ, J, (2002): Bajo el control obrero: la prensa diaria en Madrid durante la guerra civil. [Tesis Doctoral, Universidad complutense de Madrid]. Facultad de Ciencias de la Información Departamento de Historia de la Comunicación Social. Disponible en https://eprints.ucm.es/id/eprint/1854/1/T21183.pdf [Accessed 7 July 2021]

MONTORO BERMEJO, I, (2015): Libertad de Expresión e Intervención Estatal en la Radiodifusión Española de la Primera Mitad del Siglo XX [Tesis doctoral. Universidad de Murcia]. Disponible en: https://www.tdx.cat/handle/10803/311624\#page=1 (Accesed 26 May 2021)

NASEIRO RAMUDO, A, (2013): “El archivo del diario "Pueblo". Un referente para la historia de la prensa en España durante el franquismo y la transición democrática". Documentación de las Ciencias de la Información, 36, 11-29. Disponible en: <http://dx.doi.org/10.5209/rev_DCIN.2013.v36.41597> [Accessed 10 June 2021].

9 https://www.comunidad.madrid/info/cultura/form/cita-previa-archivo-regional 
PAYNE, S. (2014): Fascism in Spain, 1923-1977, Madison, University of Wisconsin Press.

REIG, R. y LANGA NUÑO, C. (2011): La comunicación en Andalucía, Sevilla, Fundación Pública Andaluza, Centro de Estudios Andaluces.

SANZ HERNANDO, C. (2017): "La prensa en el franquismo. Desarrollo y evolución historiográfica desde la dictadura a la democracia", en: PULIDO POLO, M. (editores), Tendencias de investigación en comunicación, Sevilla, Egregius, pp.1532. Disponible en: <https://dialnet.unirioja.es/servlet/libro?codigo=714748> [Accessed 6 July 2021].

SANZ HERNANDO, C. (2019): “Censura y consignas en la prensa del movimiento: el caso de "La Voz de Castilla"" en JARA CUADRADO (editores), Las huellas del franquismo: pasado y presente, Comares, pp. 1834-1854.

SINOVA, J. (1989): La censura de prensa durante el franquismo, Madrid, Espasa-Calpe.

ZALBIDEA BENGOA, B, (1996): La Prensa del Movimiento en España: 1936-1983, Bilbao, Servicio Editorial de la Universidad del País Vasco, D.L.

\section{ANEXO I}

Medios de prensa. Puntos de acceso normalizado.

7 FECHAS (SEMANARIO, MADRID, ESPAÑA, 1949-1977)

AGENCIA EFE

ALERTA (DIARIO, SANTANDER, ESPAÑA, 1937-)

AMANECER (DIARIO, ZARAGOZA, ESPAÑA, 1936-1979)

ARRIBA (DIARIO, MADRID, ESPAÑA, 1935-1979)

ARRIBA ESPAÑA (DIARIO, PAMPLONA, ESPAÑA, 1936-1975)

AYER (DIARIO, JEREZ DE LA FRONTERA, ESPAÑA, 1936-1963)

BALEARES (DIARIO, PALMA DE MALLORCA, ESPAÑA, 1939-2013)

CONSIGNA (BOLETÍN MENSUAL, LA CORUÑA, ESPAÑA, 1943-1945)

CÓRDOBA (DIARIO, CÓRDOBA, ESPAÑA, 1941-)

DIARIO ESPAÑOL (DIARIO, TARRAGONA, ESPAÑA, 1939-1984)

EL ALCÁZAR (DIARIO, MADRID, ESPAÑA, 1936-1987)

EL CORREO ESPAÑOL-EL PUEBLO VASCO (DIARIO, SAN SEBASTIAN, ESPAÑA, 1938- )

EL MERCANTIL VALENCIANO (COMUNIDAD VALENCIANA, ESPAÑA, 1872 -1939)

EL PUEBLO GALLEGO (DIARIO, VIGO, ESPAÑA, 1924-1979) 
EL SOL (DIARIO, MADRID, ESPAÑA, 1917-1939)

ESCORIAL (REVISTA, MADRID, ESPAÑA, 1940-1950)

F.E (DIARIO, SEVILLA, ESPAÑA, 1936-1946)

FALANGE (DIARIO, LAS PALMAS DE GRAN CANARIA, ESPAÑA, 1936-1963)

GOL (DIARIO, MADRID, ESPAÑA, 1938-1945)

HIERRO (DIARIO, BILBAO, ESPAÑA, 1937-1983)

IMPERIO (DIARIO, ZAMORA, ESPAÑA, 1936-1963)

INFORMACIÓN (DIARIO, ALICANTE ESPAÑA, 1941-)

JAÉN (DIARIO, JÁEN, ESPAÑA, 1941-)

JORNADA (DIARIO, VALENCIA, ESPAÑA, 1941-1975)

LA COMPAÑIA EDITORIAL ESPAÑOLA, S.A.

LA GACETA DE ALICANTE (DIARIO, ALICANTE, ESPAÑA, 1939-1941)

LA MAÑANA (DIARIO, LÉRIDA, ESPAÑA, 1938-)

LA PRENSA (DIARIO, BARCELONA, ESPAÑA, 1941-1979)

LA TARDE (DIARIO, MADRID, ESPAÑA, 1948-1949)

LA TARDE (DIARIO, MÁLAGA, ESPAÑA, 1940-1975)

LA VOZ DE CASTILLA (DIARIO, BURGOS, ESPAÑA, 1945-1976)

LA VOZ DE ESPAÑA ( DIARIO, SAN SEBASTIÁN, ESPAÑA, 1936-1980)

LA VOZ DE GUIPUZCOA (DIARIO, SAN SEBASTIAN, ESPAÑA, 1885-1936)

LA VOZ DEL SUR (SEMANARIO, CÁDIZ, ESPAÑA, 1949-1952)

LEVANTE (DIARIO, VALENCIA, ESPAÑA, 1872-)

LIBERTAD (DIARIO, VALLADOLID, ESPAÑA, 1931-1979)

LÍNEA (DIARIO, MURCIA, ESPAÑA, 1939-1983)

LOS SITIOS (DIARIO, GERONA, ESPAÑA 1943-1988)

MADRID (DIARIO, MADRID, ESPAÑA, 1939-1971)

MARAVILLAS (REVISTA, MADRID, ESPAÑA, 1939-1954)

MARCA (DIARIO, MADRID. ESPAÑA. 1938-)

MEDITERRANEO (DIARIO, CASTELLÓN DE LA PLANA, ESPAÑA, 1938-)

NUEVA ESPAÑA (DIARIO, HUESCA, ESPAÑA, 1936-1985)

ODIEL (DIARIO, HUELVA, ESPAÑA, 1935-1984)

ORDEN DE LA FALANGE (BOLETÍN MENSUAL, LA CORUÑA, ESPAÑA, 1944-1945);

PATRIA (DIARIO, GRANADA, ESPAÑA, 1935-1983)

PRENSA GRÁFICA, S.A 
Nuevas fuentes documentales para el estudio de la prensa en España durante el franquismo: el archivo regional de la Comunidad de Madrid

PRIMER PLANO, REVISTA ESPAÑOLA DE CINEMATOGRAFÍA (MADRID, ESPAÑA, 1940-1963)

PROA (DIARIO, LEÓN, ESPAÑA, 1936-1984)

PUEBLO (DIARIO, MADRID, ESPAÑA, 1940-1986)

PYRESA, Prensa y Radio Españolas, S.A.

RADIO ESPAÑA DE BILBAO

RED ESPAÑOLA DE RADIODIFUSIÓN

SEVILLA. DIARIO DE LA TARDE (DIARIO, SEVILLA, ESPAÑA, 1942-1976)

SINDICATO ESPAÑOL UNIVERSITARIO

SOLIDARIDAD NACIONAL (DIARIO, BARCELONA, ESPAÑA, 1939-1979)

SUR (DIARIO, MÁLAGA, ESPAÑA, 1937-)

TRIUNFO (SEMANARIO, VALENCIA, ESPAÑA, 1946-1982)

UNIDAD (DIARIO, SAN SEBASTIÁN, ESPAÑA, 1936-1980)

YUGO (DIARIO, ALMERÍA, ESPAÑA, 1939-1984) 